\title{
Incidencia del consumo de tóxicos en pacientes con traumatismos graves
}

\author{
M.R. DÍAZ-CONTRERASa, F. GUERRERO-LÓPEZa , L. HERRERA-PARAa, F. PINO-SÁNCHEZa, \\ R. LARA-ROSALES ${ }^{a}$, O. LÓPEZ-GUARNIDO ${ }^{b}$, A. PLA $^{\mathrm{b}}$ Y E. FERNÁNDEZ-MONDEJAR ${ }^{\mathrm{a}}$
}

aUnidad de Cuidados Intensivos. Hospital de Traumatología. Hospital Universitario Virgen de las Nieves. Granada. España. bDepartamento de Medicina Legal y Toxicología. Facultad de Medicina. Granada. España.

Objetivo. Determinar la incidencia de detección de drogas de abuso en pacientes con traumatismo grave.

Diseño. Estudio observacional prospectivo, realizado entre julio de 2004 y enero de 2006.

Ámbito. Unidad de Cuidados Intensivos (UCI) neurotraumatológica de un hospital de tercer nivel.

Pacientes. Pacientes con traumatismos que precisaron el ingreso en la $\mathrm{UCl}$ durante el período de estudio.

Intervenciones. Análisis de alcoholemia y de tóxicos (cocaína, cannabis, anfetaminas y otras) en orina.

Resultados. Se obtuvo una muestra para análisis de tóxicos en 196 pacientes de los 288 ingresados por traumatismo grave. La causa más frecuente del traumatismo fue el accidente de tráfico, con un $69 \%$. El tóxico más frecuentemente encontrado fue el cannabis $(22,4 \%)$, seguido del alcohol $(17,3 \%)$ y la cocaína $(12,8 \%)$. En menores de 45 años, el cannabis fue detectado en el $26,1 \%$ frente al $9,3 \%$ en mayores de 45 años $(p<0,05)$ y la cocaína en un $16,3 \%$ frente al $0 \%$ ( $p<0,001)$. En menores de 45 años fue detectada la presencia de algún tóxico en el $45 \%$ frente al $23 \%$ en mayores de 45 años $(p<0,05)$.

Conclusión. La alta proporción de resultados positivos a tóxicos en pacientes con traumatismos graves sugiere que el ambiente epidemiológico en que se mueven estos pacientes es muy

Correspondencia: Dra. M.R. Díaz Contreras.

$\mathrm{C} / \mathrm{Carrera}$ de la Virgen, 15.

18565 Campotéjar. Granada. España.

Correo electrónico: remedios.diaz@hotmail.com

Manuscrito aceptado el 3-XII-2007. preocupante. Estos datos deberían tener importancia para diseñar futuras campañas de prevención.

PALABRAS CLAVE: drogas de abuso, traumatismos graves, tóxicos, accidente de tráfico.

\section{INCIDENCE OF THE CONSUMPTION OF TOXIC} SUBSTANCES IN SEVERE TRAUMA PATIENTS

Objective. To determine the incidence of the detection of abuse drugs in severe trauma patients

Design. Prospective observational study conducted from July 2004 to January 2006.

Setting. Neurotrauma intensive care unit of a reference tertiary university hospital.

Patients. Trauma patients who require admission to ICU during the study period.

Interventions. Determination of alcohol in blood and of toxics (cocaine, cannabis, amphetamines and other substances) in urine.

Results. Toxicological analysis was performed in $\mathbf{1 9 6}$ of the $\mathbf{2 8 8}$ severe trauma patients admitted during the study period. The most frequently detected cause of the trauma was traffic accident (69\%). The most frequently detected substance was cannabis $(22.4 \%)$, followed by alcohol $(17.3 \%)$ and cocaine (12.8\%). Cannabis was detected in $26.1 \%$ of under-45-yr-old patients versus $9.3 \%$ of older patients ( $p<0.05$ ), and cocaine in $16.3 \%$ vs. $0 \%$ in over-45-yr-olds ( $p<0.001)$. Some substance of abuse was detected in $45 \%$ of under- $45-y r-o l d s$ versus $23 \%$ of older patients ( $p$ $<0.05$ ).

Conclusion. The high proportion of positive results to toxic substances in severely traumatized patients suggests that the epidemiological environment for these patients is of great concern. 

These data may be of interest for the design of fu-
ture prevention campaign.

KEY WORDS: drugs of abuse, severe trauma, traffic accident.

\section{INTRODUCCIÓN}

El consumo de alcohol y otras sustancias estupefacientes se puede asociar con patología subsidiaria de ingreso en la Unidad de Cuidados Intensivos (UCI) en personas jóvenes ${ }^{1,2}$. El traumatismo supone la primera causa de muerte en menores de 45 años $^{3}$; lo que unido a la posibilidad de secuelas y a la duración de la convalecencia en una franja de población particularmente productiva da idea de la trascendencia socio-sanitaria y económica de esta patología ${ }^{4,5}$.

El principal factor de riesgo para sufrir cualquier tipo de traumatismo lo constituye el consumo de sustancias que alteran el comportamiento ${ }^{6}$. En España, sólo disponemos de datos parciales en este sentido ${ }^{7}$. Los datos más fiables provienen del análisis de tóxicos realizado a personas fallecidas en accidentes de tráfico. En 2004, según el Instituto Nacional de Toxicología, el alcohol fue detectado en casi un $50 \%$ de los fallecidos en accidentes de tráfico, seguido a mucha distancia de la cocaína $(5 \%)$ y del cannabis $(1,8 \%)$, y el resto de drogas en menor porcentaje ${ }^{8}$. No disponemos, en cambio, de datos sobre la incidencia de detección de tóxicos en pacientes con traumatismos graves pero no fallecidos.

La mayor fuente de información general de la que disponemos en este campo nos llega a través de encuestas o mediante la realización de pruebas de alcoholemia realizados por agentes de tráfico en controles rutinarios. Las pruebas de alcoholemia, habitualmente mediante determinación de alcohol en aire espirado, se realizan en tres situaciones fundamentalmente: conductores implicados en accidentes de circulación, conductores que han cometido alguna infracción, y en controles de tipo preventivo9. En datos presentados por la Dirección General de Tráfico, el porcentaje de casos con alcoholemia superior a la tasa permitida en controles preventivos y analizados de forma aleatoria en el año 2002 era del 5,5\%, cifra que contrasta con la encontrada en conductores fallecidos por accidente de tráfico, que era del $32 \%$ en ese mismo año ${ }^{10,11}$. Aunque el alcohol sigue siendo el principal protagonista $^{12}$, el consumo de otras sustancias está creciendo de forma alarmante, y hay estudios que indican que el cannabis es la droga ilegal detectada con más frecuencia $^{13,14}$.

En este sentido, puede haber variaciones importantes entre los datos obtenidos en un determinado país o región y los obtenidos en otras zonas con hábitos y costumbres diferentes. En Andalucía, la prevalencia de consumo de estas sustancias en la población general, según una encuesta realizada por la Dirección General de Drogodependencias y Adicciones, revela que el $63 \%$ de la población entre 12 y
64 años ha consumido alcohol en el último mes, el $8 \%$ ha consumido cannabis y un $1,6 \%$ cocaína $^{15}$.

La importancia de la detección de pacientes que han sufrido accidentes graves relacionados con el consumo de sustancias que alteran el comportamiento viene dada no sólo por el interés epidemiológico, sino por la posibilidad de realizar una intervención que disminuya el riesgo de reincidencia. En este sentido, la detección de un accidentado relacionado con este tipo de tóxicos debe poner en marcha una estrategia de prevención secundaria tendente a evitar la reincidencia. Cuando este planteamiento se realiza, la mortalidad como consecuencia de nuevos accidentes puede reducirse significativamente ${ }^{16-19}$.

Desde 2004, en nuestra UCI estamos realizando un programa de prevención secundaria de traumatismos que aún está en curso, y cuyos resultados serán evaluados a largo plazo. En el presente trabajo, se presentan los resultados de incidencia de detección de tóxicos en pacientes con traumatismos durante un período de año y medio (del 16 julio de 2004 al 15 de enero de 2006).

\section{PACIENTES Y MÉTODO}

Se ha realizado un estudio observacional prospectivo que incluye todos los pacientes con traumatismos que ingresaron en la UCI del Hospital de Traumatología Virgen de las Nieves de Granada desde el 16 de julio de 2004 hasta el 15 de enero de 2006. El estudio fue aprobado por el Comité Ético y de Investigación de nuestro centro. No se solicitó consentimiento informado para la obtención de tóxicos, ya que, actualmente, la obtención de estas muestras se considera una parte esencial para el manejo de estos pacientes ${ }^{20}$.

De estos pacientes se obtenía una muestra de sangre para la determinación de alcoholemia mediante un cromatografía de gases, y una muestra de orina para la determinación de opiáceos, benzodiacepinas, cocaína, cannabis, metadona, anfetaminas, antidepresivos triciclícos y otros tóxicos mediante fluorescencia inmunoensayo (Triage ${ }^{\circledR}$ ). La extracción de muestras se realizaba precozmente, generalmente en las primeras 4 horas del ingreso en la UCI. El análisis de benzodiazepinas y mórficos no fue valorado, dado que la inmensa mayoría de los pacientes habían recibido este tipo de medicación en los servicios de emergencias.

Asimismo, se registraron otros datos como edad y género, mecanismo de producción del traumatismo (accidente de tráfico, laboral, agresiones y otros), la gravedad del mismo (ISS, APACHE III medido en las primeras 24 horas), días de estancia en la UCI y mortalidad, día de la semana desglosado en laboral o festivo.

Dentro de los accidentes de tráfico se contemplaba específicamente: tipo de vehículo que se había visto implicado en el accidente (coche, moto, camión y bicicleta), papel del lesionado (si era conductor, ocupaba el vehículo como acompañante o si era un viandante que había sufrido un atropello), material de 
TABLA 1. Datos demográficos y de gravedad de los 196 pacientes analizados. Resultados expresados como media \pm desviación estándar

\begin{tabular}{|lc|}
\hline Hombres & $87 \%$ \\
Edad (años) & $34,2 \pm 17,1$ \\
APACHE III (puntos) & $52,5 \pm 31,8$ \\
Injury Severity Score (puntos) & $22,6 \pm 13,4$ \\
Estancia en la UCI (días) & $9,4 \pm 11,6$ \\
Mortalidad hospitalaria & $19,40 \%$ \\
\hline
\end{tabular}

UCI: Unidad de Cuidados Intensivos.

seguridad que había utilizado (si llevaba puesto el cinturón de seguridad o el casco, según proceda), dónde se había producido el accidente (en zona urbana o en carretera).

En el análisis estadístico se utilizó la prueba de la «t» de Student o U de MannWhitney para la comparación de variables cuantitativas y la prueba de ji cuadrado para la comparación de variables cualitativas. El nivel de significación estadística se estableció en p $<0,05$.

\section{RESULTADOS}

Durante el período de estudio fueron ingresados en nuestra UCI 288 pacientes con el diagnóstico de traumatismo grave. De ellos, se obtuvo una muestra para el análisis de tóxicos en 196 (68\%). En 92 pacientes no se obtuvieron muestras, en 68 por tratarse de traslados de otros hospitales y en 24 por otros motivos. Por lo tanto, el total de pacientes analizados ha sido de 196. Los datos demográficos y de gravedad de los mismos pueden verse en la tabla 1. La causa más frecuente del traumatismo fue el accidente de tráfico (69\%), seguida de accidentes laborales (10\%), de agresiones $(9 \%)$, y otros $(12 \%)$.
La sustancia más frecuentemente encontrada fue el cannabis $(22,4 \%)$, seguida del alcohol $(17,3 \%)$ y la cocaína $(12,8 \%)$. Estos y otros resultados pueden verse en la figura 1. El nivel medio de alcoholemia detectado fue 0,98 $\pm 0,83$ gramos $/ 1$.

Salvo en el caso del alcohol, la detección de tóxicos ha sido significativamente mayor en los menores de 45 años, en comparación con los de más de 45 años. El cannabis fue detectado en el $26,1 \%$ en menores de 45 años frente al 9,3\% en mayores de 45 años, $(\mathrm{p}<0,05)$ y la cocaína en un $16,3 \%$ frente al $0 \%$ ( $\mathrm{p}<0,001)$. Quizás lo más llamativo, en este grupo de edad, sea que casi la mitad $(45,1 \%)$ de los pacientes menores de 45 años que presentan traumatismos dieron resultados positivos a algún tóxico, frente a un $23,3 \%$ en mayores de 45 años (p < 0,05) (fig. 2).

$\mathrm{Al}$ analizar la muestra según la causa del traumatismo, accidente de tráfico frente al resto (que denominaremos no tráficos), no encontramos diferencias estadísticamente significativas en el porcentaje de resultados positivos a tóxicos, aunque llama la atención la alta tasa de detección de cocaína detectada en pacientes con traumatismo por accidentes de tráfico $15,6 \%$ frente al $6,6 \%$ en los pacientes con traumatismo producido por otra causa (tabla 2 ).

Entre los que presentan traumatismo por accidente de tráfico con resultados positivos a tóxicos, el $44 \%$ eran conductores, siendo más frecuente en conductores de moto que de coche (24\% frente a 18$)$.

\section{DISCUSIÓN}

En nuestro conocimiento, estos datos son los primeros en cuantificar la incidencia de detección de tóxicos en pacientes con traumatismos graves en España. De nuestros resultados se desprende que una elevada proporción de pacientes con traumatismo, en particular los menores de 45 años, han consumido sustancias que alteran el comportamiento. El consu-

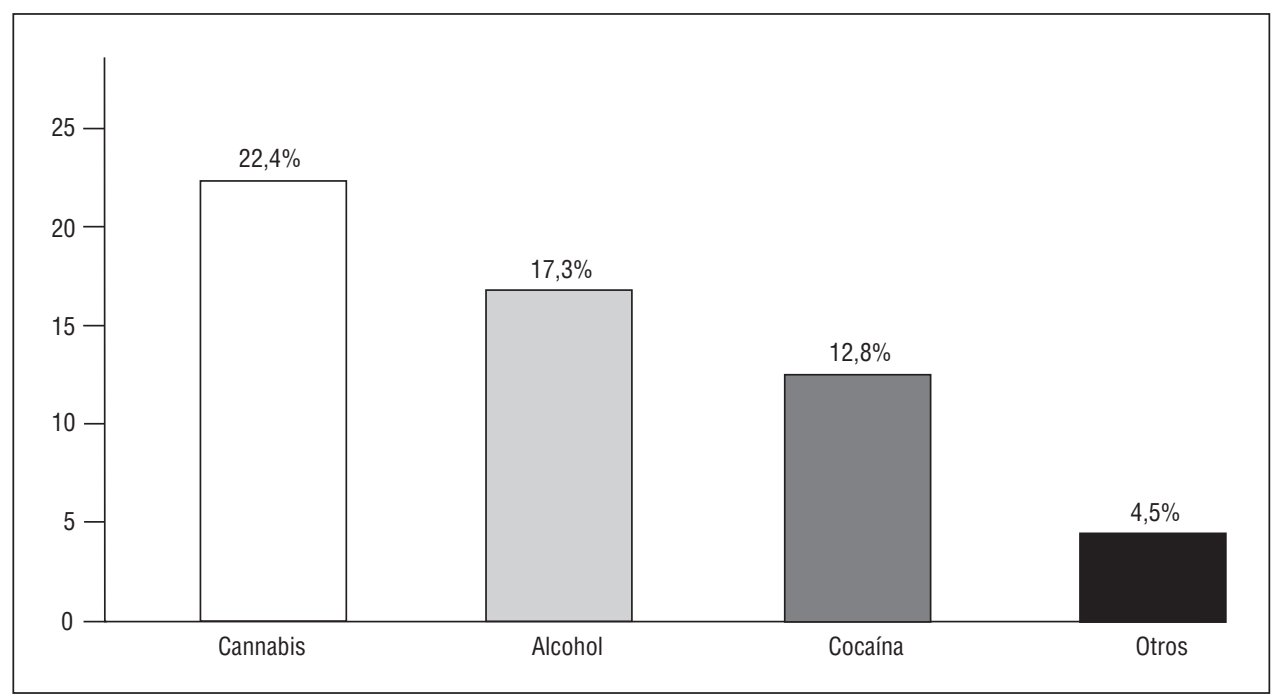

Figura 1. Presencia de tóxicos en pacientes con traumatismos. 


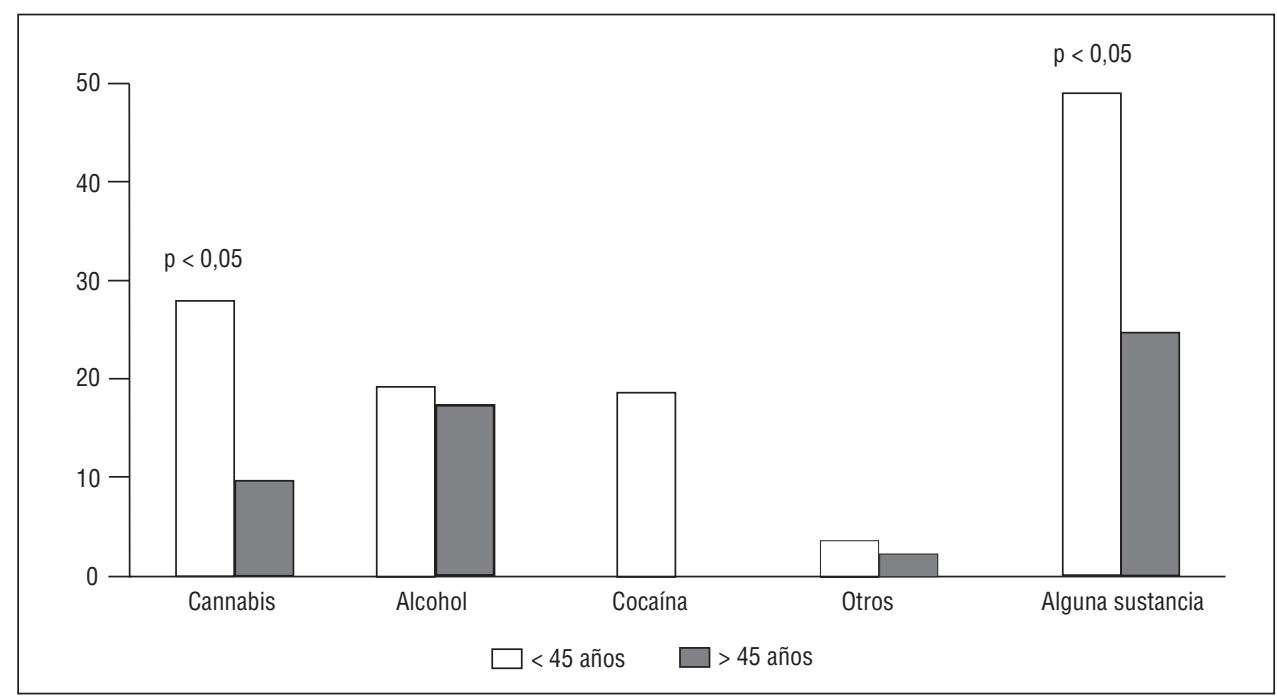

Figura 2. Consumo de drogas diferenciado en dos grupos ( $<45$ años y $>45$ años).

mo de estas sustancias puede desempeñar un papel determinante en el riesgo de sufrir accidentes y, asimismo, habla del preocupante ambiente epidemiológico en el que se mueven muchos pacientes con traumatismo.

Hasta ahora, en España sólo disponíamos de datos toxicológicos en pacientes que fallecían como consecuencia de accidentes graves, generalmente de tráfico, y la tasa de incidencia arrojaba unas cifras sensiblemente diferentes a las que nosotros hemos encontrado. En estos estudios, el alcohol es la sustancia más frecuentemente encontrada (50\%), seguida a mucha distancia de la cocaína $(5 \%)$ y del cannabis $(1,8 \%)^{6}$. Por el contrario, en nuestro estudio, la sustancia más frecuentemente encontrada ha sido el cannabis $(22,4 \%)$, seguido por el alcohol $(17,3 \%)$ y la cocaína $(12,8 \%)$. Estas cifras se incrementan sustancialmente si sólo tenemos en cuenta a los pacientes menores de 45 años (fig. 2), en este grupo, hasta un $45,1 \%$ de las personas con traumatismo habían ingerido alguna sustancia de las referidas anteriormente. Aunque no se puede establecer una relación directa causa efecto entre el traumatismo y la presencia de estas sustancias, epidemiológicamente creemos que sí existe una clara asociación, ya que la prevalencia de consumo de estas sustancias en la población general (consumo en el último mes), es muy inferior; por ejemplo, el cannabis un $8 \%$ y la cocaína un $1,6 \%$.

Hay pocos estudios internacionales que analizan la incidencia de los tóxicos en los pacientes con traumatismos graves, pero los datos que ofrecen indican una incidencia similar a la nuestra, y demuestran una tendencia a disminuir la detección de alcohol y un espectacular aumento del resto de sustancias ${ }^{6}$.

La detección de este tipo de sustancias en pacientes con traumatismos tiene trascendencia a corto plazo y largo plazo. En la fase inicial, puede ayudar en la valoración, tanto desde el punto de vista neurológico como en la orientación sobre una posible patología asociada, en particular en el caso del alcohol. También puede ayudar en la detección de una posible adicción que dificulte la evolución del paciente. Pero quizás la mayor ventaja de la detección de estas sustancias radique en la posibilidad de realizar prevención secundaria, ya que se sabe que los pacientes que han sufrido un accidente relacionado con el consumo de estas sustancias tienen el doble de posibilidades de fallecer en otro accidente que la población general ${ }^{21}$.

TABLA 2. Diferencias entre los pacientes con traumatismo por accidente de tráfico y los pacientes con traumatismo por otro motivo

\begin{tabular}{|c|c|c|c|}
\hline & $\begin{array}{l}\text { Grupo tráfico } \\
(\mathrm{n}=135)\end{array}$ & $\begin{array}{c}\text { Grupo no tráfico } \\
(\mathrm{n}=61)\end{array}$ & $\mathrm{p}$ \\
\hline Hombres & $81,60 \%$ & $86,30 \%$ & NS \\
\hline En día festivo o víspera de festivo & $60 \%$ & $36,10 \%$ & $<0,05$ \\
\hline Detección de cocaína & $15,60 \%$ & $6,60 \%$ & NS \\
\hline Detección de alcohol & $20 \%$ & $11,50 \%$ & NS \\
\hline Alcoholemia media (g/l) & 1,02 & 0,84 & NS \\
\hline Detección de cannabis & $21,40 \%$ & $24,60 \%$ & NS \\
\hline Estancia media en la UCI (días) & 9,6 & 10,8 & NS \\
\hline
\end{tabular}

NS: no significativo; UCI: Unidad de Cuidados Intensivos. 
Esto es consecuencia de la elevada tasa de reincidencia, y se ha demostrado que una adecuada intervención psicológica breve durante el encamamiento puede reducir el consumo de tóxicos, y por tanto el riesgo de nuevos accidentes ${ }^{16-19}$. La intervención psicológica breve debe realizarse durante el encamamiento, y consiste en una entrevista de unos 30 minutos tendente a poner de relieve el riesgo a que ha estado sometido, y el peligro que implica seguir con ese hábito.

Las acciones de prevención secundaria son habituales en cualquier otra patología. Actualmente, parece impensable que un paciente tratado de cáncer de pulmón, o de infarto de miocardio, no reciba consejo sobre el efecto nocivo del tabaquismo. En cambio, en el caso de los pacientes con traumatismos relacionados con el consumo de tóxicos no es habitual la realización algún tipo de prevención secundaria, a pesar de que, precisamente, el consumo de estas sustancias constituye el principal factor de riesgo para sufrir traumatismos.

No podemos descartar que la determinación de alcoholemia haya sido infraestimada, ya que, al tratarse de pacientes con traumatismos graves, generalmente sangrantes y que precisan una agresiva reposición de volemia, los niveles plasmáticos de alcohol pueden haberse visto disminuidos, o incluso ser indetectables como consecuencia de esta hemodilución. Por el contrario, la determinación de cannabis y cocaína, al ser una determinación cualitativa de metabolitos urinarios con una larga vida media ${ }^{22}$, es posible que hayamos considerado algún caso positivo de consumo previo sin relación directa con el accidente. No obstante, dada la elevada proporción de detección de estas sustancias, en comparación con los datos de prevalencia de que disponemos ${ }^{15}$, creemos que, al menos epidemiológicamente, existe una asociación manifiesta, y nuestros resultados no pueden explicarse si no es por una relación clara entre consumo y riesgo de accidente.

No hemos tenido en cuenta la presencia de benzodiacepinas ni opiáceos, dado que prácticamente el $100 \%$ de los pacientes con traumatismos reciben este tipo de medicación, suministrada por los servicios de emergencias, y dado que el análisis es cualitativo, no podemos distinguir si el paciente las había ingerido previamente. Asimismo, tan sólo hemos encontrado 3 casos $(1,5 \%)$ de resultados positivos para el consumo de anfetaminas, siendo una proporción extremadamente baja. Incluso en pacientes que reconocían haberlas ingerido, hemos obtenido resultados negativos, lo que habla de la baja sensibilidad de los reactivos actualmente disponibles en el mercado para esta sustancia. Por todo esto, consideramos que la incidencia de detección de estas sustancias ha sido sensiblemente infraestimada.

\section{AGRADECIMIENTOS}

A todo el personal, especialmente al de enfermería, de la Unidad de Medicina Intensiva Neurotraumatológica del Hospital Universitario Virgen de las Nieves de Granada.

\section{Declaración de conflicto de intereses}

Los autores han declarado no tener ningún concflicto de intereses.

\section{BIBLIOGRAFÍA}

1. Camacho Pulido JA, Jurado Lara B, Jiménez Sánchez JM, Montijano Vizcaíno A, de Molina Ortega A. Síndrome de distrés respiratorio del adulto secundario a inhalación de cocaína. Med Intensiva. 2004:28:477-8.

2. Ballesteros Herráez JC, de la Calle Gómez B, Tarancón Maján MC, Garijo Catalina MA, Rodríguez Encinas A. Miocardiopatía periparto en paciente consumidora de cocaína. Med Intensiva. 2003;27:501-3.

3. National Highway Traffic Safety Administration. Drugs and human performance fact sheet. U.S. Department of Transportation Report No. DOT HS 809 725, Washington, DC: 2004

4. Ruano M. Manual de soporte vital avanzado. 2. ${ }^{\mathrm{a}}$ ed. Barcelona: Consejo español de RCP; 2001.

5. Lucas CE. The Impact of street drugs on trauma care. J Trauma. 2005;59:S57-60.

6. Soderstrom CA, Dischinger PC, Kerns TJ, Kufera JA, Scalea TM. Epidemic increases in cocaine and opiate use by trauma center patients: documentation with a large clinical toxicology database. J Trauma. 2001;51:557-64.

7. Díaz R, Aguilar E, Herrera L, Narvona S, Guerrero F, Fernández Mondejar E. Presencia de tóxicos en pacientes traumatizados graves. Med. Intensiva. 2005;29(Número extraordinario 1); 61 .

8. Memoria de accidentes de tráfico. Madrid: Instituto Nacional de Toxicología; 2004.

9. Álvarez FJ, del Río MC. Alcohol y accidentes de tráfico: ¿prevenir qué? Valladolid: Departamento de Farmacología y Terapéutica. Facultad de Medicina. Universidad de Valladolid, 2003.

10. Memorias de accidentes de tráfico. Madrid: Instituto Nacional de Toxicología; 2002.

11. Anuario estadístico de accidentes de tráfico. Madrid: Dirección General de Tráfico; 2002.

12. Kelly E, Darke S, Ross J. A review of drug use and driving: epidemiology, impairment, risk factors and risk perceptions. Drug Alc Depend. 2004;73:319-44.

13. Walsh JM, de Gier J, Cristopherson A, Verstraete A. Drugs and driving. Traffic Inj Prev. 2004;5:241-53.

14. Merland J. Driving under the influence of non-alcoholic drugs. Forensic Sci Rev. 2000;12:80-105.

15. IX Los Andaluces ante las drogas. II Plan Andaluz sobre Drogas y Adicciones. Sevilla: Consejería para la Igualdad y Bienestar social; 2005.

16. Gentilello LM, Rivara FP, Donovan DM, Jurkovich GJ, Daranciang E, Dunn CW, et al. Alcohol interventions in a trauma center as a means of reducing the risk of injury recurrence. Ann Surg. 1999;230:473-80.

17. Zatzick D, Roy-Birne P, Russo J, Rivara F, Droesch R Wagner A, et al. A randomized effectiveness trial of stepped collaborative care for acutely injured trauma survivors. Arch Gen Psihyatry. 2004;61:498-506.

18. Hungerford DW. Interventions in trauma centers for substance use disorders: new insights on an old malady. J Trauma. 2005;59:S10-7.

19. Field C, Hungerford DW, Dunn C. Brief motivational interventions: an introduction. J Trauma. 2005;59:S21-6.

20. Gentilello LM. Alcohol and injury: American College of Surgeons Committee on trauma requirements for trauma center intervention. J Trauma. 2007;62 Suppl 6:S44-5.

21. Dischinger PC, Mitchel KA, Kufera JA, Soderstrom CA, Lowenfels AB. A longitudinal study of former trauma center patients: the assotiation between toxicology status and subsequent injury mortality. J Trauma. 2001;51:884-6.

22. González-Meneses A, Durán de Vargas LE. Consumo de drogas: marihuana, drogas de síntesis y otras. Medicina de la adolescencia. Atención integral. Madrid: Ergon; 2004. p. 200-6. 